\title{
Constant scalar curvature hypersurfaces with spherical boundary in Euclidean space
}

\section{Luis J. Alías and J. Miguel Malacarne}

Dedicated to Professor A. Gervasio Colares on the occasion of his 70th birthday

\begin{abstract}
It is still an open question whether a compact embedded hypersurface in the Euclidean space with constant mean curvature and spherical boundary is necessarily a hyperplanar ball or a spherical cap, even in the simplest case of a compact constant mean curvature surface in $\mathbb{R}^{3}$ bounded by a circle. In this paper we prove that this is true for the case of the scalar curvature. Specifically we prove that the only compact embedded hypersurfaces in the Euclidean space with constant scalar curvature and spherical boundary are the hyperplanar round balls (with zero scalar curvature) and the spherical caps (with positive constant scalar curvature). The same applies in general to the case of embedded hypersurfaces with constant $r$-mean curvature, with $r \geq 2$.
\end{abstract}

\section{Introduction.}

A classical result by Alexandrov [1] states that the spheres are the only closed embedded hypersurfaces with constant mean curvature in the Euclidean space $\mathbb{R}^{n+1}$. Here by closed hypersurfaces we mean compact and without boundary hypersurfaces. This result was extended to the case of

2000 Mathematics Subject Classification: 53A10, 53C42.

Keywords: Constant mean curvature, constant scalar curvature, constant $r$-mean curvature, Newton transformations. 
the scalar curvature by Ros in [15], where it was shown that the spheres are the only closed embedded hypersurfaces with constant scalar curvature in $\mathbb{R}^{n+1}$, solving a problem proposed by Yau in [18]. More generally, Ros [16] also extended it to the case of the $r$-mean curvature $H_{r}$, showing that the spheres are the only closed embedded hypersurfaces with constant $r$-mean curvature in $\mathbb{R}^{n+1}$.

However, when one considers the corresponding problem for the case of compact hypersurfaces with non-empty boundary, it is still unknown whether a compact embedded hypersurface in $\mathbb{R}^{n+1}$ with non-zero constant mean curvature and spherical boundary is spherical, even in the simplest case of a compact constant mean curvature surface in $\mathbb{R}^{3}$ bounded by a circle. In [10] Kapouleas showed that there exist examples of higher genus compact, non-spherical immersed surfaces with constant mean curvature in $\mathbb{R}^{3}$ bounded by a circle. It was conjectured in [9] that a compact surface with non-zero constant mean curvature in $\mathbb{R}^{3}$ bounded by a circle is a spherical cap if either the surface has genus zero and it is immersed or the surface is embedded. In the last years, different authors have considered this problem obtaining several partial results [2], [4], [5], [6], [8], but the original conjecture remains open.

In [11], Koiso gave a new interpretation of this problem by studying under what conditions the symmetries of the boundary of a non-zero constant mean curvature hypersurface $M$ embedded into $\mathbb{R}^{n+1}$ are inherited by the whole hypersurface. In particular, she showed that when the boundary $\Sigma$ is a round $(n-1)$-sphere contained in a hyperplane $\Pi$ of $\mathbb{R}^{n+1}$, and $M$ does not intersect the outside of $\Sigma$ in $\Pi$, then $M$ is symmetric with respect to every hyperplane which contains the center of $\Sigma$ and is perpendicular to $\Pi$, and hence $M$ must be a spherical cap. On the other hand, Brito, Sá Earp, Meeks and Rosenberg [9] also showed that if $\Sigma$ is a strictly convex $(n-1)$-dimensional submanifold contained in a hyperplane $\Pi$ of $\mathbb{R}^{n+1}$, and $M$ is a compact embedded hypersurface in $\mathbb{R}^{n+1}$ with constant mean curvature and $\partial M=\Sigma$ which is transverse to $\Pi$ along the boundary $\partial M$, then $M$ is contained in one of the half-spaces of $\mathbb{R}^{n+1}$ determined by $\Pi$ and $M$ has all the symmetries of $\Sigma$. More recently, Rosenberg [17] extended some of these results to the case of the $r$-mean curvatures.

In this paper we will prove that the conjecture is true for embedded hypersurfaces in the case of the scalar curvature. Specifically we will prove the following.

Theorem 1. The only compact embedded hypersurfaces in the Euclidean space with constant scalar curvature and spherical boundary are the hyperplanar round balls (with zero scalar curvature) and the spherical caps (with positive constant scalar curvature). 
In particular, for the 2-dimensional case we conclude that a compact embedded surface in $\mathbb{R}^{3}$ with constant Gaussian curvature and circular boundary is necessarily a flat planar disc or a spherical cap.

Our proof will be a consequence of more general symmetry result (Theorem 3), and it also applies in general to the case of hypersurfaces with constant $r$-mean curvature $H_{r}$, when $r \geq 2$ (Corollary 4 ). The results of this paper are part of a more general project where we derive some other new related results for compact hypersurfaces with constant higher order mean curvature in Euclidean space and, more generally, in hyperbolic and spherical spaces [3].

\section{Preliminaries.}

In this section we will review some standard facts about the geometry of Euclidean hypersurfaces. Let $\psi: M^{n} \longrightarrow \mathbb{R}^{n+1}$ be an orientable $n$-dimensional connected hypersurface immersed into the Euclidean space $\mathbb{R}^{n+1}$ (eventually with non-empty smooth boundary $\partial M$ ). Since $M$ is orientable, we may choose along $M$ a globally defined unit normal vector field $N$, and we may assume that $M$ is oriented by $N$. Let $\nabla^{\circ}$ be the flat LeviCivita connection on $\mathbb{R}^{n+1}$, and let $\nabla$ denote the Levi-Civita connection on $M$. Then the Gauss and Weingarten formulae for the hypersurface are given by

$$
\nabla_{X}^{\mathrm{o}} Y=\nabla_{X} Y+\langle A X, Y\rangle N
$$

and

$$
A(X)=-\nabla_{X}^{\mathrm{o}} N
$$

for all tangent vector fields $X, Y \in \mathcal{X}(M)$. Here $A: \mathcal{X}(M) \longrightarrow \mathcal{X}(M)$ defines the shape operator (or the second fundamental form) of the hypersurface with respect to $N$. The curvature tensor $R$ of the hypersurface $M$ is described in terms of $A$ by the Gauss equation, which is given by

$$
R(X, Y) Z=\langle A X, Z\rangle A Y-\langle A Y, Z\rangle A X,
$$

for all tangent vector fields $X, Y, Z \in \mathcal{X}(M)$. Observe that our criterion here for the definition of the curvature tensor is the one in [13].

As is well known, $A$ is a self-adjoint linear operator, and its eigenvalues $\kappa_{1}, \ldots, \kappa_{n}$ are the principal curvatures of the hypersurface. Associated to the shape operator there are $n$ algebraic invariants, which are the elementary symmetric functions of its eigenvalues, given by

$$
S_{r}=S_{r}\left(\kappa_{1}, \ldots, \kappa_{n}\right)=\sum_{i_{1}<\cdots<i_{r}} \kappa_{i_{1}} \cdots \kappa_{i_{r}}, \quad 1 \leq r \leq n .
$$


Observe that the characteristic polynomial of $A$ can be written in terms of the $S_{r}$ 's as

$$
\operatorname{det}(t I-A)=\sum_{r=0}^{n}(-1)^{r} S_{r} t^{n-r} .
$$

The $r$-mean curvature $H_{r}$ of the hypersurface is then defined by

$$
\left(\begin{array}{l}
n \\
r
\end{array}\right) H_{r}=S_{r}
$$

In particular, when $r=1$ then $H_{1}=(1 / n) \operatorname{trace}(A)=H$ is the mean curvature of $M$, which is the main extrinsic curvature of the hypersurface. On the other hand, when $r=2, H_{2}$ defines a geometric quantity which is related to the (intrinsic) scalar curvature of the hypersurface. Indeed, from (1) we know that the Ricci curvature of $M$ is given by

$$
\operatorname{Ric}(X, Y)=n H\langle A X, Y\rangle-\langle A X, A Y\rangle,
$$

for $X, Y \in \mathcal{X}(M)$, so that its scalar curvature $S$ is

$$
S=\operatorname{trace}(\mathrm{Ric})=n(n-1) H_{2} .
$$

In general, it follows from the Gauss equation (1) that when $r$ is odd $H_{r}$ is extrinsic (and its sign depends on the chosen orientation), while when $r$ is even $H_{r}$ is an intrinsic geometric quantity.

The classical Newton transformations $P_{r}: \mathcal{X}(M) \longrightarrow \mathcal{X}(M)$ are defined inductively from $A$ by

$$
P_{0}=I \quad \text { and } \quad P_{r}=S_{r} I-A P_{r-1}, \quad 1 \leq r \leq n,
$$

where $I$ denotes the identity in $\mathcal{X}(M)$, or equivalently by

$$
P_{r}=S_{r} I-S_{r-1} A+\cdots+(-1)^{r-1} S_{1} A^{r-1}+(-1)^{r} A^{r} .
$$

Note that by the Cayley-Hamilton theorem, we have $P_{n}=0$. Let us recall that each $P_{r}$ is also a self-adjoint linear operator which commutes with $A$. Indeed, $A$ and $P_{r}$ can be simultaneously diagonalized; if $\left\{e_{1}, \ldots, e_{n}\right\}$ are the eigenvectors of $A$ corresponding to the eigenvalues $\kappa_{1}, \ldots, \kappa_{n}$, respectively, then they are also the eigenvectors of $P_{r}$ corresponding to the eigenvalues of $P_{r}$, and $P_{r}\left(e_{i}\right)=\mu_{i, r} e_{i}$ with

$$
\mu_{i, r}=\frac{\partial S_{r+1}}{\partial \kappa_{i}}=\sum_{i_{1}<\cdots<i_{r}, i_{j} \neq i} \kappa_{i_{1}} \cdots \kappa_{i_{r}}, \quad 1 \leq i \leq n .
$$


From here it can be easily seen that

$$
\operatorname{trace}\left(P_{r}\right)=(n-r) S_{r}=c_{r} H_{r}
$$

and

$$
\operatorname{trace}\left(A P_{r}\right)=(r+1) S_{r+1}=c_{r} H_{r+1}
$$

where

$$
c_{r}=(n-r)\left(\begin{array}{l}
n \\
r
\end{array}\right)=(r+1)\left(\begin{array}{c}
n \\
r+1
\end{array}\right) .
$$

For the details, we refer the reader to the classical paper by Reilly [14] (see also [17] for a more accesible modern treatment by Rosenberg).

\section{A geometric configuration.}

Throughout this paper, we will consider the following geometric configuration in Euclidean space $\mathbb{R}^{n+1}$. Let $\Pi \subset \mathbb{R}^{n+1}$ be a hyperplane in $\mathbb{R}^{n+1}$, and let $\Sigma^{n-1} \subset \Pi$ be an orientable $(n-1)$-dimensional compact submanifold contained in $\Pi$. Let $\psi: M^{n} \longrightarrow \mathbb{R}^{n+1}$ be an orientable compact hypersurface immersed into $\mathbb{R}^{n+1}$ with smooth boundary $\partial M$. As usual, $M$ is said to be a hypersurface with boundary $\Sigma$ if the immersion $\psi$ restricted to the boundary $\partial M$ is a diffeomorphism onto $\Sigma$. In order to orient this geometric configuration, let us consider the hypersurface $M$ oriented by a globally defined unit normal vector field $N$. The orientation of $M$ induces a natural orientation on its boundary as follows: given a point $p \in \partial M$, a basis $\left\{v_{1}, \ldots, v_{n-1}\right\}$ for $T_{p}(\partial M)$ is said to be positively oriented if $\left\{u, v_{1}, \ldots, v_{n-1}\right\}$ is a positively oriented basis for $T_{p} M$, whenever $u \in T_{p} M$ is outward pointing. We will denote by $\nu$ the outward pointing unit conormal vector field along $\partial M$. By means of the diffeomorphism $\left.\psi\right|_{\partial M}: \partial M \longrightarrow \Sigma$, the orientation of $\partial M$ is induced on each connected component of $\Sigma$. On the other hand, let $\eta$ be the unitary vector field normal to $\Sigma$ in $\Pi$ which points outward with respect to the domain in $\Pi$ bounded by $\Sigma$. Now, we choose $a$ the unique unitary vector field normal to $\Pi$ which is compatible with $\eta$ and with the orientation of $\Sigma$. With this choice, given a point $p \in \Sigma$, a basis $\left\{v_{1}, \ldots, v_{n-1}\right\}$ for $T_{p} \Sigma$ is positively oriented if and only if $\left\{\eta(p), v_{1}, \ldots, v_{n-1}\right\}$ is a positively oriented basis for II.

Let $\left\{e_{1}, \ldots, e_{n-1}\right\}$ be a (locally defined) positively oriented frame field along $\partial M$. Using this frame, we can write $\nu=e_{1} \times \cdots \times e_{n-1} \times N$, and 
similarly $\eta=e_{1} \times \cdots \times e_{n-1} \times a$, since $\operatorname{det}\left(\nu, e_{1}, \ldots, e_{n-1}, N\right)=1=$ $\operatorname{det}\left(\eta, e_{1}, \ldots, e_{n-1}, a\right)$. From these expressions we easily find that

$$
\langle\eta, N\rangle=-\langle a, \nu\rangle
$$

Let $A_{\Sigma}$ denote the shape operator of $\Sigma^{n-1} \subset \Pi$ with respect to the unit normal vector field $\eta$. It then follows that

$$
\nabla_{e_{i}}^{\mathrm{o}} e_{j}=\sum_{k=1}^{n-1}\left\langle\nabla_{e_{i}}^{\mathrm{o}} e_{j}, e_{k}\right\rangle e_{k}+\left\langle\nabla_{e_{i}}^{\mathrm{o}} e_{j}, \nu\right\rangle \nu+\left\langle A e_{i}, e_{j}\right\rangle N
$$

for every $1 \leq i, j \leq n-1$, and also

$$
\nabla_{e_{i}}^{\mathrm{o}} e_{j}=\sum_{k=1}^{n-1}\left\langle\nabla_{e_{i}}^{\mathrm{o}} e_{j}, e_{k}\right\rangle e_{k}+\left\langle A_{\Sigma} e_{i}, e_{j}\right\rangle \eta,
$$

so that from (5) we have that

$$
\left\langle A e_{i}, e_{j}\right\rangle=-\left\langle A_{\Sigma} e_{i}, e_{j}\right\rangle\langle a, \nu\rangle, \quad 1 \leq i, j \leq n-1 .
$$

We now suppose that the basis $\left\{e_{1}, \ldots e_{n-1}\right\}$ on the boundary is chosen such that, at the point $p \in \partial M$, it is formed by the eigenvectors of $A_{\Sigma}$, and let us denote its corresponding eigenvalues by $\tau_{1}, \ldots, \tau_{n-1}$. In other words,

$$
A_{\Sigma} e_{i}=\tau_{i} e_{i}, \quad 1 \leq i \leq n-1 .
$$

Hence by (6), $\left\langle A e_{i}, e_{j}\right\rangle=0$ when $i \neq j$, and for each $p \in \partial M$, the matrix of $A$ in the orthonormal basis $\left\{e_{1}, \ldots e_{n-1}, \nu\right\}$ of $T_{p} M$ is given by

$$
A=\left(\begin{array}{ccccc}
-\tau_{1}\langle a, \nu\rangle & 0 & \cdots & 0 & \left\langle A \nu, e_{1}\right\rangle \\
0 & -\tau_{2}\langle a, \nu\rangle & \cdots & 0 & \left\langle A \nu, e_{2}\right\rangle \\
\vdots & \vdots & \ddots & \vdots & \vdots \\
0 & 0 & \cdots & -\tau_{n-1}\langle a, \nu\rangle & \left\langle A \nu, e_{n-1}\right\rangle \\
\left\langle A \nu, e_{1}\right\rangle & \left\langle A \nu, e_{2}\right\rangle & \cdots & \left\langle A \nu, e_{n-1}\right\rangle & \langle A \nu, \nu\rangle
\end{array}\right)
$$

This expression invites us to compute the characteristic polynomial of $A$. To do that, we begin by observing that for each $k, 1 \leq k \leq n-1$,

$$
\begin{aligned}
& \operatorname{det}\left(t I_{k+1}-\Lambda_{k}\right) \\
& \quad=\left(t+\tau_{k}\langle a, \nu\rangle\right) \operatorname{det}\left(t I_{k}-\Lambda_{k-1}\right)-\left\langle A \nu, e_{k}\right\rangle^{2} \prod_{j=1}^{k-1}\left(t+\tau_{j}\langle a, \nu\rangle\right),
\end{aligned}
$$


where $\Lambda_{k}=\Lambda\left(\tau_{1}, \ldots, \tau_{k}\right)$ is the $(k+1)$-dimensional matrix given by

$$
\Lambda_{k}=\left(\begin{array}{ccccc}
-\tau_{1}\langle a, \nu\rangle & 0 & \cdots & 0 & \left\langle A \nu, e_{1}\right\rangle \\
0 & -\tau_{2}\langle a, \nu\rangle & \cdots & 0 & \left\langle A \nu, e_{2}\right\rangle \\
\vdots & \vdots & \ddots & \vdots & \vdots \\
0 & 0 & \cdots & -\tau_{k}\langle a, \nu\rangle & \left\langle A \nu, e_{k}\right\rangle \\
\left\langle A \nu, e_{1}\right\rangle & \left\langle A \nu, e_{2}\right\rangle & \cdots & \left\langle A \nu, e_{k}\right\rangle & \langle A \nu, \nu\rangle
\end{array}\right) .
$$

In particular, $\Lambda_{n-1}=A$ and therefore, applying a simple induction argument in (8), we obtain that the characteristic polynomial of $A$ is given by

$$
\begin{aligned}
\operatorname{det}\left(t I_{n}-A\right)= & (t-\langle A \nu, \nu\rangle) \prod_{i=1}^{n-1}\left(t+\tau_{i}\langle a, \nu\rangle\right) \\
& -\sum_{i=1}^{n-1}\left\langle A \nu, e_{i}\right\rangle^{2} \prod_{j=1, j \neq i}^{n-1}\left(t+\tau_{j}\langle a, \nu\rangle\right) \\
= & (t-\langle A \nu, \nu\rangle)\left(t^{n-1}+\sum_{i=1}^{n-1} s_{i}\langle a, \nu\rangle^{i} t^{n-1-i}\right) \\
& -\sum_{i=1}^{n-1}\left\langle A \nu, e_{i}\right\rangle^{2}\left(t^{n-2}+\sum_{j=1}^{n-2} s_{j}\left(\widehat{\tau}_{i}\right)\langle a, \nu\rangle^{j} t^{n-2-j}\right),
\end{aligned}
$$

where $s_{r}$ (respectively $s_{r}\left(\widehat{\tau}_{i}\right)$ ) stands for the elementary symmetric functions of $\tau_{1}, \ldots, \tau_{n-1}$, (respectively $\tau_{1}, \ldots, \widehat{\tau}_{i}, \ldots, \tau_{n-1}$ ), and, as usual, $s_{0}=$ $s_{0}\left(\widehat{\tau_{i}}\right)=1$ by definition. Comparing the terms of above polynomials, we conclude from (2) that the $r$-mean curvature $H_{r}$ of the hypersurface $M$, at a boundary point $p \in \partial M$, is given by

$$
n H_{1}=S_{1}=-s_{1}\langle a, \nu\rangle+\langle A \nu, \nu\rangle
$$

$$
\begin{aligned}
\left(\begin{array}{l}
n \\
2
\end{array}\right) H_{2}=S_{2} & =s_{2}\langle a, \nu\rangle^{2}-s_{1}\langle a, \nu\rangle\langle A \nu, \nu\rangle-\sum_{i=1}^{n-1}\left\langle A \nu, e_{i}\right\rangle^{2} \\
\left(\begin{array}{l}
n \\
r
\end{array}\right) H_{r}=S_{r}= & (-1)^{r} s_{r}\langle a, \nu\rangle^{r}+(-1)^{r-1} s_{r-1}\langle a, \nu\rangle^{r-1}\langle A \nu, \nu\rangle \\
& +(-1)^{r-1}\langle a, \nu\rangle^{r-2} \sum_{i=1}^{n-1} s_{r-2}\left(\widehat{\tau}_{i}\right)\left\langle A \nu, e_{i}\right\rangle^{2},
\end{aligned}
$$


for $3 \leq r \leq n$, where $s_{n}=0$ by definition.

Now we are ready to prove the following essential auxiliary result.

Lemma 2. Let $\Sigma$ be an orientable $(n-1)$-dimensional compact submanifold contained in a hyperplane $\Pi=a^{\perp}$ of $\mathbb{R}^{n+1}$. Let $\psi: M^{n} \longrightarrow \mathbb{R}^{n+1}$ be an orientable hypersurface with boundary $\Sigma=\psi(\partial M)$, and let $\nu$ stands for the outward pointing unit conormal vector field along $\partial M \subset M$. Then, along the boundary $\partial M$ and for every $1 \leq r \leq n-1$, it holds

$$
\left\langle P_{r} \nu, \nu\right\rangle=(-1)^{r} s_{r}\langle a, \nu\rangle^{r}
$$

where $s_{r}$ is the $r$-th symmetric function of the principal curvatures of $\Sigma \subset$ $\Pi$ with respect to the outward pointing unitary normal.

Proof. We will use induction on $r$. Observe that for $r=1$, we have that

$$
\left\langle P_{1} \nu, \nu\right\rangle=S_{1}-\langle A \nu, \nu\rangle
$$

which jointly with (9) implies

$$
\left\langle P_{1} \nu, \nu\right\rangle=-s_{1}\langle a, \nu\rangle
$$

For a given $2 \leq r \leq n-1$, suppose that

$$
\left\langle P_{j} \nu, \nu\right\rangle=(-1)^{j} s_{j}\langle a, \nu\rangle^{j}
$$

holds for all $1 \leq j \leq r-1$. Observe that

$$
A \nu=\sum_{i=1}^{n-1}\left\langle A \nu, e_{i}\right\rangle e_{i}+\langle A \nu, \nu\rangle \nu
$$

so that from the inductive definition of $P_{r}$ and (11) we conclude that

$$
\begin{aligned}
\left\langle P_{r} \nu, \nu\right\rangle & =S_{r}-\left\langle P_{r-1} \nu, A \nu\right\rangle \\
& =S_{r}-\left\langle P_{r-1} \nu, \nu\right\rangle\langle A \nu, \nu\rangle-\sum_{i=1}^{n-1}\left\langle P_{r-1} \nu, e_{i}\right\rangle\left\langle A \nu, e_{i}\right\rangle \\
& =S_{r}+(-1)^{r} s_{r-1}\langle a, \nu\rangle^{r-1}\langle A \nu, \nu\rangle-\sum_{i=1}^{n-1}\left\langle P_{r-1} \nu, e_{i}\right\rangle\left\langle A \nu, e_{i}\right\rangle
\end{aligned}
$$


On the other hand, we also know from (7) that

$$
A e_{i}=-\tau_{i}\langle a, \nu\rangle e_{i}+\left\langle A \nu, e_{i}\right\rangle \nu
$$

so that from our induction hypothesis (11) we have for every $1 \leq j \leq r-1$,

$$
\begin{aligned}
\left\langle P_{j} \nu, e_{i}\right\rangle & =-\left\langle P_{j-1} \nu, A e_{i}\right\rangle \\
& =\tau_{i}\langle a, \nu\rangle\left\langle P_{j-1} \nu, e_{i}\right\rangle-(-1)^{j-1} s_{j-1}\langle a, \nu\rangle^{j-1}\left\langle A \nu, e_{i}\right\rangle .
\end{aligned}
$$

This implies by a recursive argument that

$$
\begin{aligned}
\left\langle P_{r-1} \nu, e_{i}\right\rangle & =(-1)^{r-1}\left\langle A \nu, e_{i}\right\rangle\langle a, \nu\rangle^{r-2} \sum_{j=0}^{r-2}(-1)^{j} s_{r-2-j} \tau_{i}^{j} \\
& =(-1)^{r-1}\left\langle A \nu, e_{i}\right\rangle\langle a, \nu\rangle^{r-2} s_{r-2}\left(\widehat{\tau}_{i}\right)
\end{aligned}
$$

since it is not difficult to see that

$$
s_{m}\left(\widehat{\tau}_{i}\right)=\sum_{j=0}^{m}(-1)^{j} s_{m-j} \tau_{i}^{j},
$$

for every $1 \leq m \leq n-1$. Using now (13) in (12), along with (9), we conclude that

$$
\begin{aligned}
\left\langle P_{r} \nu, \nu\right\rangle= & S_{r}+(-1)^{r} s_{r-1}\langle a, \nu\rangle^{r-1}\langle A \nu, \nu\rangle \\
& +(-1)^{r}\langle a, \nu\rangle^{r-2} \sum_{i=1}^{n-1} s_{r-2}\left(\widehat{\tau}_{i}\right)\left\langle A \nu, e_{i}\right\rangle^{2} \\
= & (-1)^{r} s_{r}\langle a, \nu\rangle^{r} .
\end{aligned}
$$

This finishes the proof of Lemma 2 .

\section{Proof of the main results.}

Our Theorem 1 will be a consequence of the following symmetry result, which extends a previous result given by Rosenberg [17, Theorem 7.3] (observe that the case $r=n$ was first proved by Rosenberg).

Theorem 3. Let $\Sigma$ be an strictly convex compact $(n-1)$-dimensional submanifold contained in a hyperplane $\Pi \subset \mathbb{R}^{n+1}$, and let $\psi: M^{n} \longrightarrow \mathbb{R}^{n+1}$ 
be an embedded compact hypersurface with boundary $\Sigma$. Let us assume that for a given $2 \leq r \leq n$, the $r$-mean curvature $H_{r}$ of $M$ is a non-zero constant. Then $M$ is contained in one of the half-spaces of $\mathbb{R}^{n+1}$ determined by $\Pi$ and $M$ has all the symmetries of $\Sigma$.

Proof. By choosing an appropriate orientation of $M$, we may assume without loss of generality that $H_{r}$ is a positive constant. Actually, it is not difficult to see that under the hypothesis above there exists at least one interior elliptic point of $M$, that is, an interior point of $M$ where all the principal curvatures are positive. In fact, since $M$ is not part of a hyperplane (because of $H_{r} \neq 0$ ), then one easily finds a radius $R>0$ and a point $a \in \mathbb{R}^{n+1}$ such that the closed round ball $\bar{B}(a, R)$ contains $M$ and such that there is a point $p_{0} \in \operatorname{int}(M) \cap \partial B(a, R)$ (englobe $M$ with spheres of large radius until such a sphere touches $M$ on one side at an interior point). This fact, jointly with the fact that $H_{r}$ is a positive constant, allows us to conclude that the Newton transformation $P_{r-1}$ is positive definite on $M$ (see [7, Proposition 3.2] and [17, p. 232]). In particular, by (10) it follows that $\langle a, \nu\rangle(p) \neq 0$ at every boundary point $p \in \partial M$, what means that $M$ is transverse to $\Pi$ along the boundary. Our result then is a consequence of [17, Theorem 7.3].

Now Theorem 1 easily follows from Theorem 3 . Indeed, if $M$ is not a hyperplanar round ball, then the constant scalar curvature, or equivalently $\mathrm{H}_{2}$ (see equation (3)), must be necessarily positive because there exists at least one interior elliptic point of $M$. The same applies in general for the case of hypersurfaces with constant $r$-mean curvature $H_{r}$, when $r \geq 2$.

Corollary 4. The only embedded compact hypersurfaces in the Euclidean space with constant $r$-mean curvature $H_{r}$ (with $2 \leq r \leq n$ ) and spherical boundary are the hyperplanar round balls (with $H_{r}=0$ ) and the spherical caps (with $H_{r}$ a non-zero constant).

Acknowledgements. This paper is part of the $\mathrm{Ph}$. D. Thesis of the second author [12], which was defended in Universidade Federal do Ceará, Fortaleza (Brazil), in February 2001. He would like to thank his adviser, Prof. Luquésio P. M. Jorge, for his guidance.

This work was started while the first author was visiting the Institute des Hautes Études Scientifiques at Bures-sur-Yvette (France) in Summer 2000. He would like to thank that institution for their wonderful hospitality. 
The authors are grateful to Prof. H. Rosenberg and Prof. A. Ros for their interest and for providing several useful comments on this paper to improve it.

\section{References.}

[1] Alexandrov, A. D., Uniqueness theorems for surfaces in the large V. Vestnik Leningrad Univ. Math. 13 (1958), 5-8. English translation: A.M.S. Transl. 21 (1962), 412-416.

[2] Alías, L. J., López, R., PAlmer, B., Stable constant mean curvature surfaces with circular boundary. Proc. Amer. Math. Soc. 127 (1999), 1195-1200.

[3] Alías, L. J., Herbert S. De Lira, J., Malacarne, J. M., Constant higher order mean curvature hypersurfaces in Riemannian spaces. Preprint, 2002.

[4] Alías, L. J., Palmer, B., On the area of constant mean curvature discs and annuli with circular boundaries. Math. Z. 237 (2001), 585-599.

[5] Barbosa, J. L., Constant mean curvature surfaces bounded by a planar curve. Mat. Contemp. 1 (1991), 3-15.

[6] Barbosa, J. L., Hypersurfaces of constant mean curvature in $\mathbb{R}^{n+1}$ bounded by an Euclidean sphere. Geometry and topology of submanifolds, II, 1-9. World Sci. Publishing, 1990.

[7] Barbosa, J. L., Colares, A. G., Stability of hypersurfaces with constant $r$-mean curvature. Ann. Global Anal. Geom. 15 (1997), 277-297.

[8] Barbosa, J. L., Jorge, L. P., Stable $H$-surfaces spanning $\mathbb{S}^{1}(1) A n$. Acad. Brasil. Cienc. 61 (1994), 259-263.

[9] Brito, F., Sá Earp, R., Meeks, W., Rosenberg, H., Structure theorems for constant mean curvature surfaces bounded by a planar curve. Indiana Univ. Math. J. 40 (1991), 333-343.

[10] Kapouleas, N., Compact constant mean curvature surfaces in Euclidean three-space. J. Differential Geom. 33 (1991), 683-715.

[11] KoIso, M., Symmetry of hypersurfaces of constant mean curvature with symmetric boundary. Math. Z. 191 (1986), 567-574.

[12] Malacarne, J. M., Fórmulas do fluxo, simetrias e $r$-curvatura média constante. Ph. D. Thesis. Universidade Federal do Ceará, Fortaleza, Brazil, 2001.

[13] O'NeILL, B., Semi-Riemannian geometry with applications to relativity. Academic Press, 1983.

[14] Reilly, R. C., Variational properties of functions of the mean curvature for hypersurfaces in space forms. J. Differential Geom. 8 (1973), 465-477. 
[15] Ros, A., Compact hypersurfaces with constant scalar curvature and a congruence theorem. J. Differential Geom. 27 (1988), 215-220.

[16] Ros, A., Compact hypersurfaces with constant higher order mean curvatures. Rev. Mat. Iberoamericana 3 (1987), 447-453.

[17] Rosenberg, H., Hypersurfaces of constant curvature in space forms. Bull. Sci. Math. 117 (1993), 211-239.

[18] YAu, S. T., Problem section. Seminar on Differential Geometry. Annals Math. Studies No. 102. Princeton University Press, 1982.

Recibido: 7 de marzo de 2001

Luis J. Alías*

Departamento de Matemáticas

Universidad de Murcia E-30100 Espinardo, Murcia, SPAIN ljalias@um.es

and

J. Miguel Malacarne ${ }^{\dagger}$ Departamento de Matemática Universidade Federal do Espírito Santo 29060-900 Vitória-ES, BRAZIL jmiguel@cce.ufes.br

* Partially supported by DGICYT, MECD, Spain (BPM2001 2871-C04-02) and by Fundación Séneca, CARM, Spain (PI-3/00854/FS/01).

$\dagger$ Partially supported by PICD/CAPES, Brazil. 